\title{
EFFECT OF HEAT TREATMENT ON THE STRUCTURE AND PHASE COMPOSITION OF ALUMINUM MATRIX COMPOSITES CONTAINING SILICON CARBIDE
}

\author{
N. B. Pugacheva*, I. Yu. Malygina, N. S. Michurov, \\ E. I. Senaeva, N. P. Antenorova \\ Institute of Engineering Science, Ural Branch of the Russian Academy of Sciences, \\ 34 Komsomolskaya St., Ekaterinburg, Russia \\ *Corresponding author. E-mail: nat@imach.uran.ru \\ Address for correspondence: ul. Komsomolskaya, 34, Ekaterinburg, Russian Federation \\ Tel.: +7 (343) 37535 91; fax: +7 (343) 3745330
}

The paper studies the microstructure, phase and chemical compositions and hardness of composites with a D16 alloy matrix and $\mathrm{SiC}$ as a filler in the amounts of 10, 20 and $30 \%$ after quenching from $540{ }^{\circ} \mathrm{C}$ followed by aging at temperatures of 120 and $170{ }^{\circ} \mathrm{C}$. It has been found that the matrix has a grain structure, the grain size being determined by the size of the granules; namely, the coarser the granules ( 5 to $150 \mu \mathrm{m}$ ), the larger the grains $(0.05$ to $5 \mu \mathrm{m}$, respectively). Needleshaped particles, up to $2 \mu \mathrm{m}$ long and $0.5 \mu \mathrm{m}$ wide, of the $S$-phase of $\mathrm{Al}_{2} \mathrm{CuMg}$ precipitate on the matrix grain boundaries. At a quenching temperature of $540{ }^{\circ} \mathrm{C}$, all the reinforcing intermetallics dissolve in the metal matrix, with the formation of low-melting eutectics according to the reaction $\alpha+S\left(\mathrm{Al}_{2} \mathrm{CuMg}\right) \rightarrow L$; eutectic structural constituents of two chemical compositions are formed one including the copper- and zinc-enriched S-phase, the other containing magnesium. Herewith, the melt flows into the micropores among the filler particles, this being manifested especially clearly in the composite with $30 \mathrm{vol} \% \mathrm{SiC}$. Silicon carbide partially dissolves with the formation of $\mathrm{Al}_{4} \mathrm{SiC}_{4}$. Hardness measurements demonstrate that, after sintering, in the initial composites there are internal micropores at the interfaces of three and more $\mathrm{SiC}$ filler particles, and this decreases hardness from $107 \mathrm{HV} 5$ at $5 \% \mathrm{SiC}$ to $71.6 \mathrm{HV} 5$ at $30 \% \mathrm{SiC}$. Heating and holding at $540{ }^{\circ} \mathrm{C}$ increases the values of hardness due to lower porosity, these values being further increased by aging at 120 and $170{ }^{\circ} \mathrm{C}$. It is proposed that heating to $540{ }^{\circ} \mathrm{C}$ be used to perform heat-deformation processing of the studied composites after sintering in order to decrease porosity, to ensure strong diffusion bonding of the matrix to the filler particles and to form the most homogeneous possible structure, aging at 120 or $170{ }^{\circ} \mathrm{C}$ being used for the final hardening of finished products.

Keywords: composite, matrix, filler, eutectics, quenching, aging, intermetallics, hardness

DOI: $10.17804 / 2410-9908.2017 .6 .028-036$

\section{References}

1. Fernández R., González-Doncel G. Understanding the creep fracture behavior of aluminum alloys and aluminum alloy metal matrix composites. Materials Science and Engineering: A, 2011, vol. 528, iss. 28, pp. 8218-8225. DOI: 10.1016/j.msea.2011.07.027

2. Yishi S., Qiubao O., Wenlong Z., Zhiqiang L., Qiang G., Genlian F., Di Z. Composite structure modeling and mechanical behavior of particle reinforced metal matrix composites. Mater. Sci. Eng. A., 2014, vol. 597, pp. 359-369. DOI: 10.1016/j.msea.2014.01.024

3. Zhao Long-Zhi, Zhao Ming-Juan, Yan Hong, Cao Xiao-Ming, Zhang Jin-Song. Mechanical behavior of $\mathrm{SiC}$ foam-SiC particles/Al hybrid composites. Transactions of Nonferrous Metals Society of China, 2009, vol. 19, pp. 547-551. DOI: 10.1016/S10036326(10)60106-9

4. $\quad$ Ortega-Celaya F., Pech-Canul M.I., Lopes-Cuevars J., Rendon-Angeles J.C., Pech-Canul M.A. Microstructure and impact behavior of $\mathrm{Al} / \mathrm{SiCp}$ composites fabricated by pressureless infiltration 
with different types of SiCp. Journal of Materials Processing Technology, 2007, vol. 183, nos. 2-3, pp. 368-373. DOI: 10.1016/j.jmatprotec.2006.10.029

5. Konovalov A.V., Smirnov S.V. The state of the art and lines of research in the field of $\mathrm{Al} / \mathrm{SiC}$ metal matrix composites. Konstruktsii iz Kompozitsionnykh Materialov, 2015, no. 1, pp. 30-35. (In Russian).

6. Pugacheva N.B., Michurov N.S., Bikova T.M. The Structure and Properties of the 30Al-70SiC Metal Matrix Composite Material. Diagnostics, Resource and Mechanics of materials and structures, 2015, iss. 6, pp. 6-18. Available at: http://dreamjournal.org/issues/2015-6/2015-6_56.html 11

7. Pugacheva N.B., Michurov N.S., Bykova T.M. Structure and properties of the Al/SiC composite material. Physics of Metals and Metallography, 2016, vol. 117, no. 6, pp. 634-640. DOI: $10.1134 / \mathrm{S} 0031918 X 16060119$

8. Smirnov A.S., Konovalov A.V., Muizemnek O.Yu. Modelling and Simulation of Strain Resistance of Alloys Taking into Account Barrier Effects. Diagnostics, Resource and Mechanics of materials and structures, 2015, iss. 1, pp. 61-72. Available at: http:// dreamjournal.org/issues/2015-1/2015-1_18.html

9. Khalevitsky Yu.V., Myasnikova M.V., Konovalov A.V. Ways of creating a computational model of the representative volumes an $\mathrm{Al} / \mathrm{SiC}$ metal matrix composite with an internal structure. Matematicheskoe Modelirovanie v Estestvennykh Naukakh, 2014, vol. 1, pp. 277-280. (In Russian). 10. Kolachev B.A., Yelagin V.I., Livanov V.A. Metallovedenie i termicheskaya obrabotka metallov [Metal Science and Heat Treatment of Metals]. M, MISIS Publ., 2001, 416 p. (In Russian).

11. Fridlyander I.N. Modern Aluminum and Magnesium Alloys and Composite Materials Based on Them. Metal Science and Heat Treatment, 2002, vol. 44, iss 7, pp. 292-296. DOI: 10.1023/A: 1021255804324

12. Lee Doh-Jae, Vaudin M.D., Handewerker C.A., Katter U.R. Phase Stability and Interface Reactions in the Al-SiC System. In: Mater. Res. Symp. Proc., 1988, vol. 120, pp. 293. DOI: $10.1557 /$ PROC-120-357

13. Ibrahim A.I., Mohamed F.A., Lavernia E.J. Particular reinforced metal matrix composites a review. Journal of Materials Science, 1991, vol. 26, iss. 5, pp. 1137-1156. DOI: 10.1007/BF00544448

14. Pugacheva N.B., Michurov N.S., Senaeva E.I. Structure and thermophysical properties of aluminum-matrix composites. Physics of Metals and Metallography, 2016, no. 11, pp. 11441151. DOI: $10.1134 / \mathrm{S} 0031918 X 16110119$ 
Подана в журнал: 03.11.2017

УДК 620.186 .1

DOI: $10.17804 / 2410-9908.2017 .6 .028-036$

\title{
ВЛИЯНИЕ ТЕРМООБРАБОТКИ НА СТРУКТУРУ И ФАЗОВЫЙ СОСТАВ АЛЮМОМАТРИЧНЫХ КОМПОЗИТОВ С КАРБИДОМ КРЕМНИЯ
}

\author{
Н. Б. Пугачева*, И. Ю. Малыгина, Н. С. Мичуров, \\ Е. И. Сенаева, Н. П. Антенорова
}

\begin{abstract}
Федеральное государственное бюджетное учреждение науки Институт машиноведения Уральского отделения Российской академии наук, Екатеринбург, Российская Федерация

*Ответственный автор. Электронная почта: nat@imach.uran.ru Адрес для переписки: 620049, Екатеринбург, ул. Комсомольская, 34, Российская Федерация Тел.: +7 (343) 375-35-91; факс: +7 (343) 374-53-30
\end{abstract}

Исследована микроструктура, фазовый и химический составы и твердость композитов с матрицей из сплавов Д16 с содержанием наполнителя - карбида кремния $\mathrm{SiC}-5,10,20$ и $30 \%$ после закалки от $540{ }^{\circ} \mathrm{C}$ и последующего старения при температурах 120 и $170{ }^{\circ} \mathrm{C}$. Установлено, что матрица в исследованных композитах имеет зеренное строение, причем размер зерен определяется размерами гранул: чем крупнее гранулы (от 5 до 150 мкм), тем больше размер зерна (от 0,05 до 5 мкм) соответственно. Определено, что по границам зерен металлической матрицы выделяются частицы $S$-фазы $\mathrm{Al}_{2} \mathrm{CuMg}$ игольчатой формы, длина частиц достигает 2 мкм при ширине 0,5 мкм. При температуре $540{ }^{\circ} \mathrm{C}$ происходит растворение всех упрочняющих интерметаллидов в металлической матрице и образуется легкоплавкая эвтектика по реакции $\alpha+S\left(\mathrm{Al}_{2} \mathrm{CuMg}\right) \rightarrow L$, после охлаждения которой формируются эвтектические структурные составляющие двух химических составов: первый включает $S$-фазу, обогащенную медью и цинком, а второй - магнием. При этом происходит затекание расплава в микропоры между частицами наполнителя, что особенно ярко проявилось в композите с 30 об. \% SiC. При этом происходит частичное растворение карбида кремния с образованием соединения $\mathrm{Al}_{4} \mathrm{SiC}_{4}$. Замеры твердости показали, что в исходных композитах после спекания присутствуют внутренние микропоры на участках стыка трех и более частиц наполнителя $\mathrm{SiC}$, что сказывается на уменьшении твердости от $107 \mathrm{HV} 5$ при 5 \% SiC до 71,6 HV 5 при 30 \% SiC. Нагрев и выдержка при температуре $540{ }^{\circ} \mathrm{C}$ привели к повышению значений твердости за счет снижения пористости, а старение при 120 и $170{ }^{\circ} \mathrm{C}$ дополнительно увеличило эти значения. Предложено использовать нагрев до $540{ }^{\circ} \mathrm{C}$ для проведения термодеформационной обработки исследованных композитов после спекания для снижения пористости, установления прочных диффузионных связей между матрицей и частицами наполнителя, а также формирования максимально однородной структуры, а старение при 120 или 170 ${ }^{\circ} \mathrm{C}$ - для окончательной упрочняющей обработки готовых изделий.

Ключевые слова: композит, матрица, наполнитель, эвтектика, закалка, старение, интерметаллиды. твердость.

\section{1. Введение}

Композиционные материалы с алюминиевой матрицей в последние годы привлекают пристальное внимание исследователей и конструкторов. Это обусловлено особыми свойствами этих композитов, а именно, сочетанием высоких прочностных характеристик, хорошей технологической обрабатываемости при относительно низкой стоимости [1-5]. В качестве металлической матрицы используют технический алюминий или такие алюминиевые сплавы, как АМг6, В95, Д16 и др. Эти сплавы характеризуются малой плотностью, высокой 
электропроводностью, удовлетворительной коррозионной стойкостью. Если при анализе алюмоматричных композитов (АМК) с матрицей из технического алюминия или термически неупрочняемых сплавов (таких как АМг6) на первое место выступают соотношение размера матричных гранул и частиц наполнителя $\mathrm{SiC}$ [6-9], то при использовании термически упрочняемых сплавов, таких как В95 и Д16, весьма важно структурное состояние матрицы, определяемое режимом термической обработки. Для АМК с матрицей из термически упрочняемых сплавов появляется дополнительная возможность повышения прочностных свойств или улучшения технологической обрабатываемости за счет проведения упрочняющей термической обработки, заключающейся в закалке и старении $[10,11]$. До сих пор исследования алюмоматричных композитов (АМК) касались в основном методов их получения и оценки структуры и свойств. Все больший интерес вызывают работы, посвященные исследованиям специальных технологических обработок (термической и термо-деформационной) на изменение структуры, фазового состава и свойств АМК. В связи с этим актуальным является исследование влияния термической обработки на изменения структуры, фазового и химического составов АМК с матрицей из термически упрочняемых сплавов и карбидам кремния $\mathrm{SiC}$ в качестве наполнителя.

\section{2. Материалы и методы исследования}

Исследована микроструктура, фазовый и химический составы, твердость композитов с матрицей из сплавов Д16 и с содержанием наполнителя - карбида кремния $\mathrm{SiC}-5,10,20$ и 30 \% после термической обработки по следующим режимам: 1) нагрев до $540{ }^{\circ} \mathrm{C}$, выдержка 15 мин, закалка в воде; 2) нагрев до $540{ }^{\circ} \mathrm{C}$, выдержка 15 мин, закалка в воде; старение $120{ }^{\circ} \mathrm{C} 24$ ч; 3) нагрев до $540{ }^{\circ} \mathrm{C}$, выдержка 15 мин, закалка в воде; старение $170{ }^{\circ} \mathrm{C} 10$ ч. Для получения композитов использовали гранулированный сплав Д16 следующего химического состава, мас. \%: до 0,3 Zn; $1,2-1,8 \mathrm{Mg} ; 3,8-4,9 \mathrm{Cu}$; до 0,5 Fe; до 0,5 Si; 0,3-0,9 Mn; 0,1-0,25 Cr; до $0,5 \mathrm{Ni}$ до $0,1 \mathrm{Ti}, \mathrm{Al}$ - остальное. Композиты получены методом спекания по технологии ВИАМ (г. Москва). В дальнейшем для получения изделий требуемых размеров и формы спеченные композиты подвергают деформационной обработке, которая чаще всего проводится при повышенных температурах, обеспечивающих твердорастворное состояние матрицы. Проведение операции закалки имело две цели: 1) растворить все интерметаллиды в матрице и сформировать пересыщенный твердый раствор легирующих элементов в ГЦК-решетке алюминия для получения максимальной пластичности композитов, необходимой для реализации деформационно-термической обработки заготовок; 2) увеличить прочность связей матрицы с наполнителем за счет их диффузионного взаимодействия в условиях образования легкоплавких эвтектик, которые, как известно $[12,13]$, могут образовываться в сплаве при температурах $480{ }^{\circ} \mathrm{C}$ и выше. Старение проводили для подтверждения возможности упрочнения матрицы частицами $T-\left(\mathrm{Mg}_{3} \mathrm{Zn}_{3} \mathrm{Al}_{2}\right) ; \eta-\left(\mathrm{MgZn}_{2}\right)$ и $S-\left(\mathrm{Al}_{2} \mathrm{CuMg}\right) ; \mathrm{Al}_{4} \mathrm{SiC}_{4}$ и $\mathrm{Al}_{4} \mathrm{C}_{3}$, которое следует рекомендовать в качестве заключительного этапа технологической обработки готового изделия.

Микроструктуру АМК исследовали на поперечных шлифах с помощью оптического микроскопа NEOPHOT-21. Фазовый состав определяли на рентгеновском дифрактометре Shimadzu XRD 7000 в монохроматизированном К $\alpha$ излучении хромового анода в узком интервале дифракционных максимумов с максимальной интенсивностью для фаз $T-\left(\mathrm{Mg}_{3} \mathrm{Zn}_{3} \mathrm{Al}_{2}\right), \eta-\left(\mathrm{MgZn}_{2}\right)$, $S-\left(\mathrm{Al}_{2} \mathrm{CuMg}\right), \mathrm{Al}_{4} \mathrm{SiC}_{4}$ и $\mathrm{Al}_{4} \mathrm{C}_{3}$ при скорости вращения гониометра 0,25 град./мин. Локальный химический состав в точке диаметром 1 мкм или на участке поверхности размерами $100 \times 100$ мкм определяли на растровом электронном микроскопе TESCAN VEGAII XMU (Чехия) с волнодисперсионной и энергодисперсионной приставками фирмы OXFORD, оснащенными программным обеспечением INCA. Значения твердости АМК определяли методом Виккерса на приборе Akashy при нагрузке 5 кг. Кроме того, дополнительные сведения о фазовом составе получали при анализе линий Кикучи методом дифракции обратно-

Pugacheva N.B. et al. / Effect of heat treatment on the structure and phase composition of aluminum matrix composites containing silicon carbide 
отраженных электронов при помощи приставки Oxford NKLNordlysF+ с программным комплексом HKL Channel 5.

\section{3. Обсуждение результатов}

Известно [10,11], что в термически упрочняемых алюминиевых сплавах, таких как сплавы Д16 и В95, происходят следующие эвтектические реакции: при температу$\operatorname{pax} 380-480{ }^{\circ} \mathrm{C} \mathrm{L} \rightarrow \alpha+T\left(\mathrm{Mg}_{3} \mathrm{Zn}_{3} \mathrm{Al}_{2}\right)$ и/или $\mathrm{L} \rightarrow \alpha+\eta\left(\mathrm{MgZn}_{2}\right)$, а при более высокой температуре $530-540{ }^{\circ} \mathrm{C} \mathrm{L} \rightarrow \alpha+S\left(\mathrm{Al}_{2} \mathrm{CuMg}\right)$. Частицы $\eta-$ и $T$-фаз имеют размеры порядка 20-50 нм и равномерно распределены по всему объему сплава, они наблюдаются только методом просвечивающей электронной микроскопии. Для исследуемых АМК приготовить фольгу для такого анализа невозможно, поскольку при электрополировке частицы наполнителя $\mathrm{SiC}$ будут выкрашиваться, при этом делая края образовавшихся «дырок» не просвечивающими для пучка электронов. Установить присутствие частиц $\eta$ - и $T$-фаз возможно рентгеноструктурным методом только при малых скоростях вращения гониометра, чтобы выделить дифракционные максимумы максимальной интенсивности из линий фона. С этой целью все образцы исследовали в узких интервалах углов, соответствующих наиболее сильным дифракционным максимумам искомых фаз.

Частицы $S$-фазы $\mathrm{Al}_{2} \mathrm{CuMg}$ имеют игольчатую форму, длина частиц достигает 2 мкм при ширине - 0,5 мкм. Выделяются частицы $S$-фазы по границам зерен алюминиевой матрицы (рис. 1). Размер зерна зависит от размера гранул матрицы: чем больше размер гранулы, тем крупнее зерно в ней. Размер гранул матрицы составляет от 10 до 150 мкм, а размеры зерна меняются от 0,05 до 5 мкм. Наличие $S$-фазы по границам зерен матрицы зафиксировано микрорентгеноспектральным анализом и при анализе линий Кикучи для этой фазы в обратно-рассеянных электронах. Кроме того, $S$-фаза выявлена при рентгеноструктурном анализе.

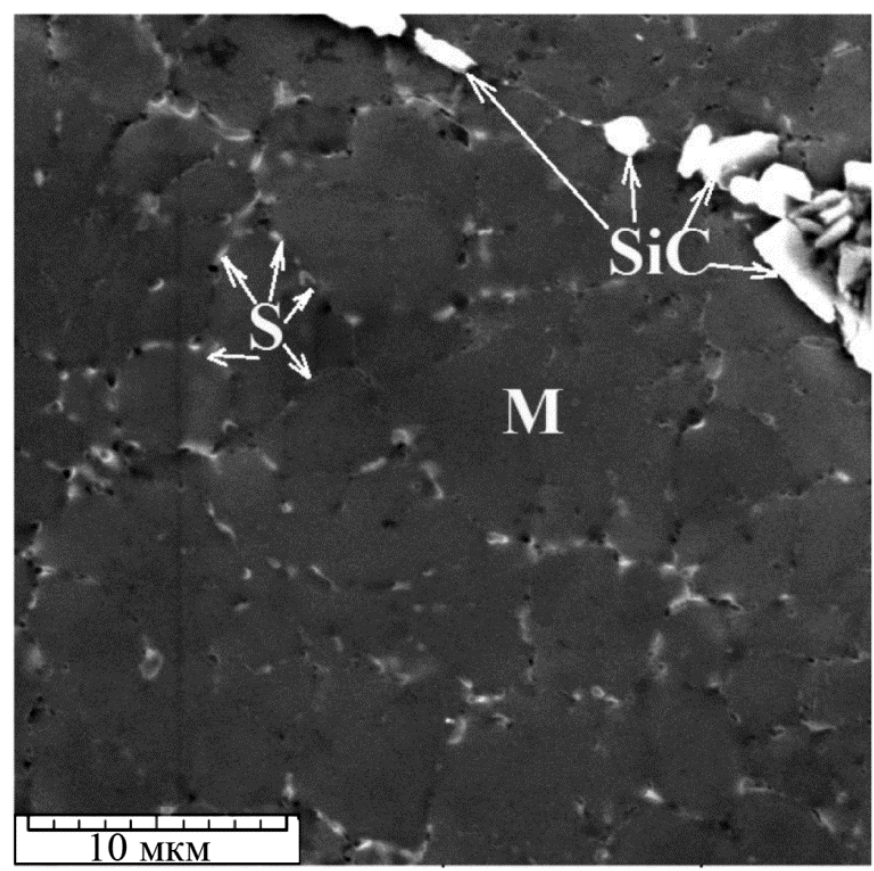

Рис. 1. Микроструктура исследованных алюмоматричных композитов: $\mathrm{M}$ - матрица; $\mathrm{S}$ - частицы интерметаллидов $\mathrm{Al}_{2} \mathrm{CuMg}$

Ранее экспериментально было установлено, что перед началом плавления композитов в матрице из сплава В95, близкого по химическому составу сплаву Д16, происходит эндо-

Pugacheva N.B. et al. / Effect of heat treatment on the structure and phase composition of aluminum matrix composites containing silicon carbide 
термическое превращение (рис. 2). Выполненный в данной работе металлографический анализ показал, что при температуре выдержки $540{ }^{\circ} \mathrm{C}$, которая несколько выше температуры зафиксированного эндотермического превращения, происходит локальное оплавление, причем более развито оно по границам зерен матрицы по реакции: $\alpha+S\left(\mathrm{Al}_{2} \mathrm{CuMg}\right) \rightarrow L$. Таким образом, для композитов с матрицей из сплава Д16 также характерно эндотермическое превращение, подобно установленному нами ранее [14].

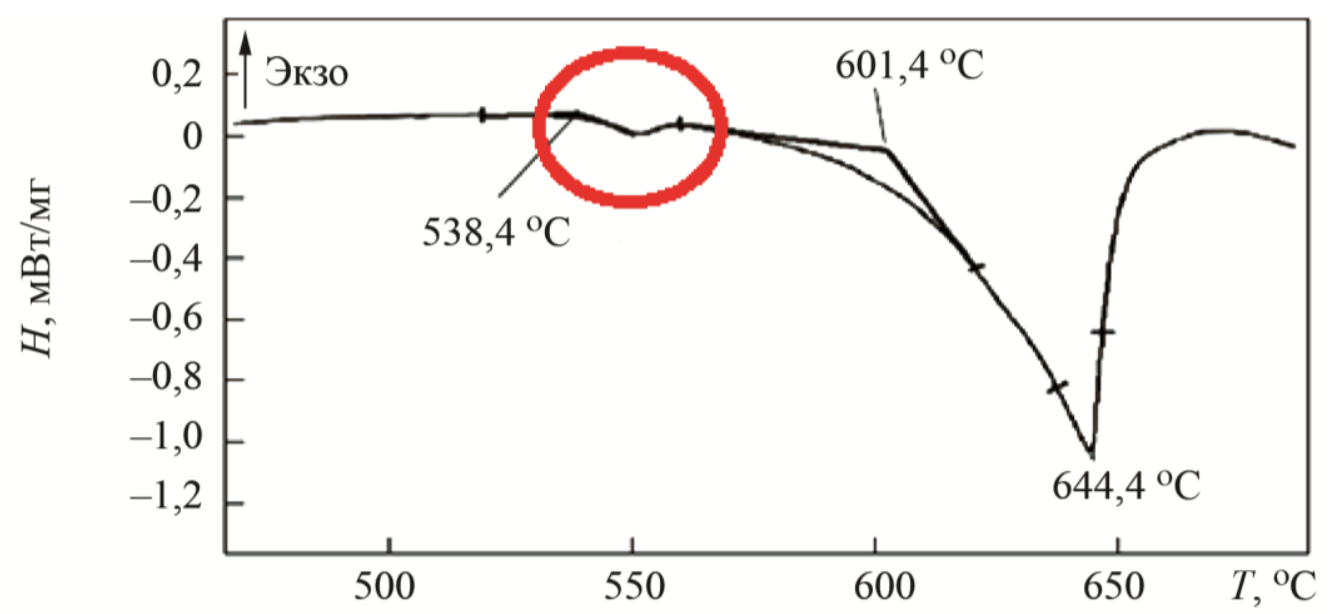

Рис. 2. Пример ДСК-кривой для композита с матрицей В95 и 20 об. \% $\mathrm{SiC}$ [14]

Экспериментально было установлено, что в исследованных АМК присутствует эвтектика двух составов: первый включает $S$-фазу, обогащенную медью и цинком, а второй - магнием (рис. 3). Появление жидкой фазы вблизи частиц наполнителя облегчает растворение карбида кремния $\mathrm{SiC}$, хотя согласно диаграмме состояния системы $\mathrm{Al}-\mathrm{SiC}[12,13]$ эвтектическое превращение в ней происходит при более высокой температуре $578{ }^{\circ} \mathrm{C}$. Частичное растворение $\mathrm{SiC}$ в расплаве экспериментально зафиксировано при микрорентгеноспектральном анализе: в эвтектике обнаружен кремний в количествах до 12 мас. \%, причем в эвтектике, обогащенной магнием содержание кремния в 2-3 раза выше, чем в эвтектике, обогащенной медью и цинком. Образовавшийся при $540{ }^{\circ} \mathrm{C}$ расплав проникает между частицами $\mathrm{SiC}$, что способствует «залечиванию» микропор на участках стыка в трех и более частиц наполнителя $\mathrm{SiC}$.

Выполненный фазовый рентгеноструктурный анализ в узком интервале углов при малой скорости вращения гониометра зафиксировал в $\mathrm{AMK}$ следующие фазы: $\mathrm{Al}_{4} \mathrm{SiC}_{4}$ на всех исследованных образцах, а также $\eta-\left(\mathrm{MgZn}_{2}\right)$ и $S-\left(\mathrm{Al}_{2} \mathrm{CuMg}\right)$ на всех образцах после старения при температуре $170{ }^{\circ} \mathrm{C}$. Тем не менее, значения твердости после старения при $120^{\circ} \mathrm{C}$ увеличилось на $25 \%$, что свидетельствует о дисперсионном твердении матрицы. Очевидно, при этой температуре выделяются более дисперсные частицы, дифракционные максимумы которых не удалось выделить из линии фона из-за слишком малых областей когерентного рассеяния.

Измерение значений твердости показало, что с увеличением содержания наполнителя в композитах в исходном состоянии происходит уменьшение твердости на $25 \%$. Это обусловлено увеличением количества внутренних пор в местах контакта трех и более частиц наполнителя $\mathrm{SiC}$. Проведение выдержки при температуре $540{ }^{\circ} \mathrm{C}$ в течение 15 мин привело к частичному залечиванию пор путем затекания в них расплава, при этом образование эвтектических структур при охлаждении до комнатной температуры не снизило значение твердости (см. таблицу). 


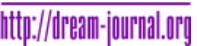

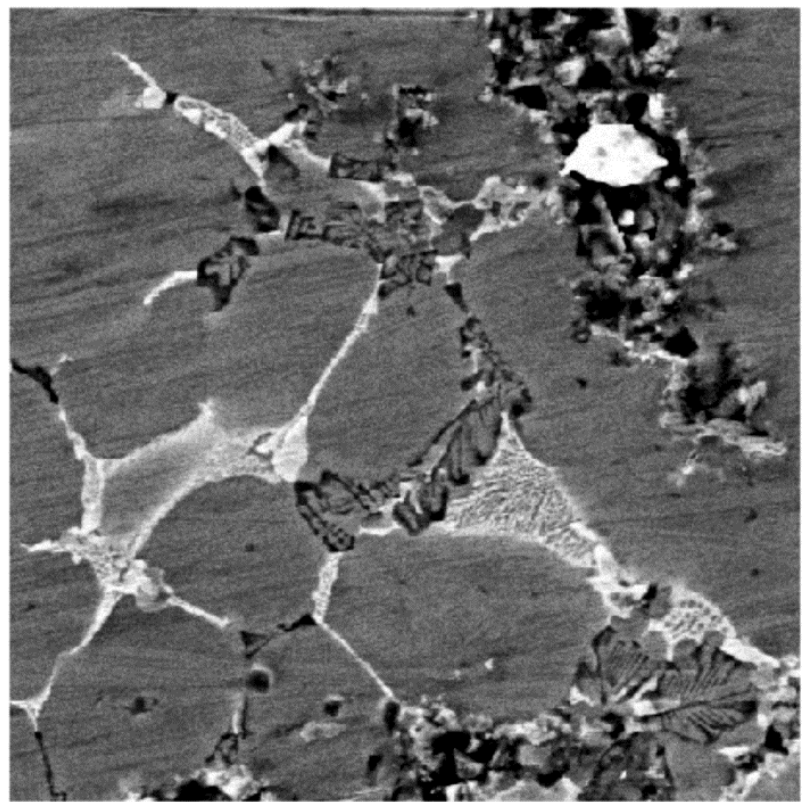

$a$

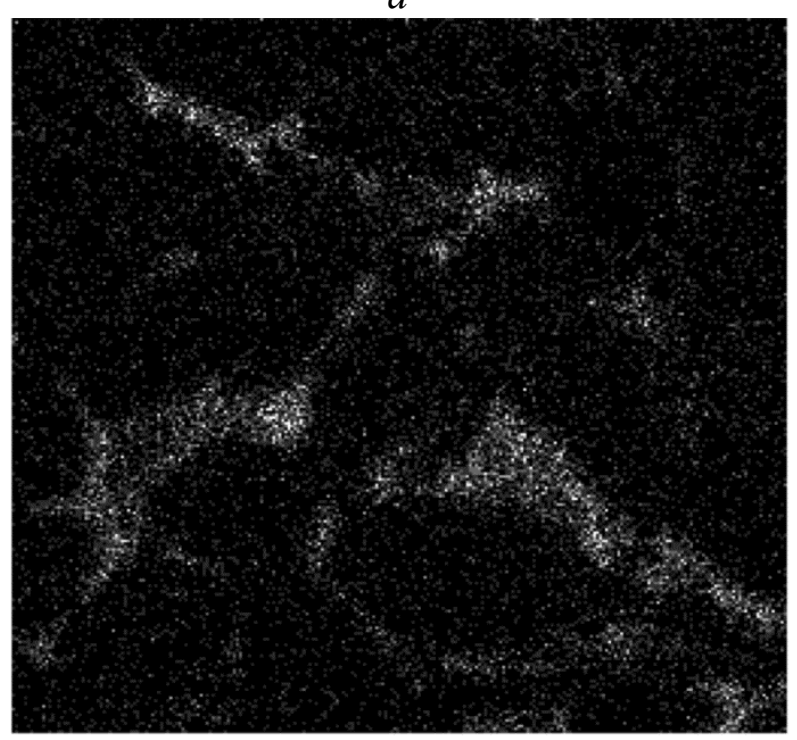

$\mathrm{Cu} \mathrm{Kal}$

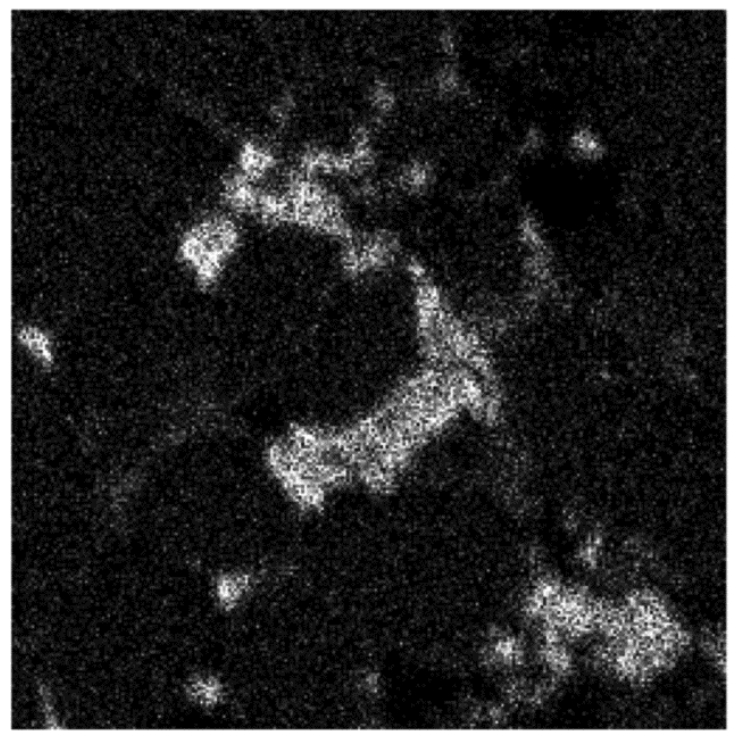

Mg Kal_2

6

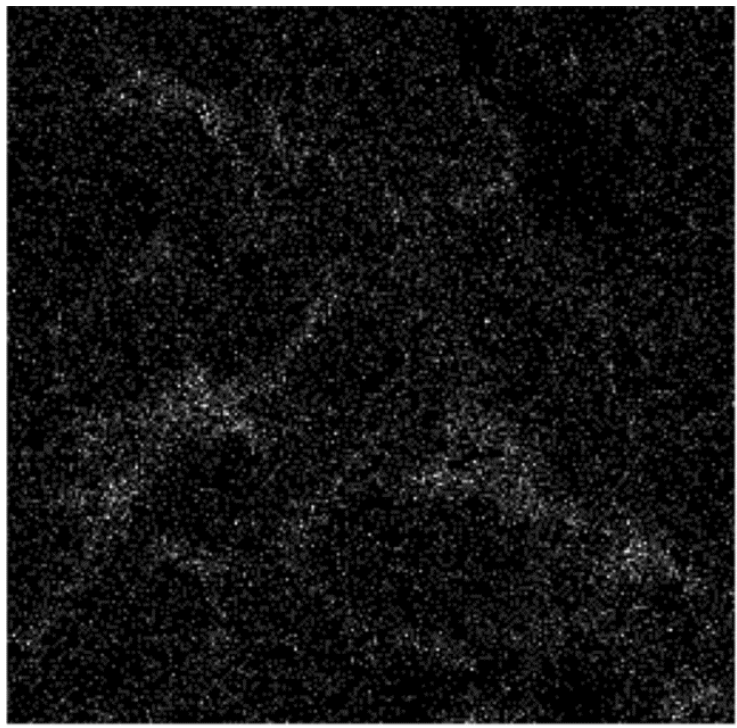

Zn Kal

B

2

Рис. 3. Распределение элементов в эвтектике, образовавшейся при нагреве и выдержке при $540{ }^{\circ} \mathrm{C}$ в композите Д16+30\% SiC

Изменение твердости композитов после термической обработки, HV5

\begin{tabular}{|c|c|c|c|c|c|}
\hline \multirow{2}{*}{ № } & \multirow{2}{*}{$\begin{array}{c}\text { Содержание } \\
\text { наполнителя } \\
\text { SiC, об. \% }\end{array}$} & $\begin{array}{c}\text { Заготовка } \\
\text { после } \\
\text { спекания }\end{array}$ & $\begin{array}{c}\text { Закалка } \\
540^{\circ}, \\
15 \text { мин, вода }\end{array}$ & $\begin{array}{c}\text { Закалка + старение } \\
120^{\circ} \mathrm{C}, 24 \text { ч }\end{array}$ & $\begin{array}{c}\text { Закалка + старение } \\
170^{\circ} \mathrm{C}, 10 \text { ч }\end{array}$ \\
\hline 1 & 5 & 107 & 101 & 127 & 110 \\
\hline 2 & 10 & 95,3 & 110 & 125 & 109 \\
\hline 3 & 20 & 95,3 & 105 & 127 & 96,5 \\
\hline 4 & 30 & 71,6 & 95,3 & 58,8 & \\
\hline
\end{tabular}

Pugacheva N.B. et al. / Effect of heat treatment on the structure and phase composition of aluminum matrix composites containing silicon carbide 
Полученные результаты позволяют предложить температуру $540{ }^{\circ} \mathrm{C}$ для использования при термо-деформационной обработке спеченных заготовок из исследованных композитов после их спекания. Такая обработка позволит устранить внутреннюю микропористость композитов за счет затекания образовавшегося расплава в существующие микропоры, а также обеспечит формирование прочного диффузионного взаимодействия матрицы с частицами наполнителя с образованием промежуточного химического соединения $\mathrm{Al}_{4} \mathrm{SiC}_{4}$. Необходимы дальнейшие дополнительные исследования влияния степени деформации при различных температурах на структурное состояние АМК, позволяющие выбрать наиболее благоприятные условия деформирования не только с целью залечивания пор, но и с точки зрения разбиения скелетообразных эвтектических структур.

\section{4. Выводы}

Показано, что матрица из гранулированного сплава Д16 в исследованных АМК имеет зеренное строение, причем размер зерен определяется размерами гранул: чем крупнее гранулы, тем больше размер зерна. Размер гранул матрицы составляет от 10 до 150 мкм, а размеры зерна меняются от 0,05 до 5 мкм. По границам зерен металлической матрицы при медленном охлаждении из однофазной области сплава Д16 или после старения выделяются частицы $S$-фазы $\mathrm{Al}_{2} \mathrm{CuMg}$ игольчатой формы, длина частиц достигает 2 мкм при ширине 0,5 мкм.

Установлено, что при температуре $540{ }^{\circ} \mathrm{C}$ происходит растворение всех упрочняющих интерметаллидов в металлической матрице исследованных АМК, а также образуется легкоплавкая эвтектика по реакции $\alpha+S\left(\mathrm{Al}_{2} \mathrm{CuMg}\right) \rightarrow L$. В результате после охлаждения в воде образуются эвтектические структурные составляющие двух химических составов: первый включает $S$-фазу, обогащенную медью и цинком, а второй - магнием.

Образование легкоплавкой эвтектики в исследованных композитах при температуре выдержки $540{ }^{\circ} \mathrm{C}$ приводит к затеканию расплава в микропоры между частицами наполнителя, что особенно ярко проявилось в композите с 30 об. \% SiC. При этом происходит частичное растворение карбида кремния с образованием соединения $\mathrm{Al}_{4} \mathrm{SiC}_{4}$, присутствие которого зафиксировано при рентгеноструктурном анализе с малой скоростью вращения гониометра.

Замеры твердости показали, что в исходных спеченных композитах присутствуют внутренние микропоры на участках стыка трех и более частиц наполнителя SiC, что сказывается на уменьшении твердости от $107 \mathrm{HV} 5$ у композита с 5 \% SiC до 71,6 HV 5 у композита с $30 \% \mathrm{SiC}$. Закалка от температуры $540{ }^{\circ} \mathrm{C}$ привела к повышению значений твердости за счет снижения пористости, а старение дополнительно увеличило эти значения.

Полученные результаты позволяют предложить температуру $540{ }^{\circ} \mathrm{C}$ для проведения термо-деформационной обработки исследованных композитов после их спекания для снижения пористости, установления прочных диффузионных связей между матрицей и частицами наполнителя, а также формирования максимально однородной структуры.

\section{Благодарность}

Работа выполнена при частичной поддержке проекта РНФ № 14-19-01358 (в области исследованных материалов).

\section{Литература}

1. Fernandez R., Gonzalez-Doncel G. Understanding the creep fracture behavior of aluminum alloys and aluminum alloy metal matrix composites // Mater. Sci. Eng. A. - 2011. - Vol. 528. P. 8218-8225. - DOI: 10.1016/j.msea.2011.07.027

2. Composite structure modeling and mechanical behavior of particle reinforced metal matrix composites / S. Yishi, O. Qiubao, Z. Wenlong, L. Zhiqiang, G. Qiang, F. Genlian, Z. Di // Mater. Sci. Eng. A. - 2014. - Vol. 597. - P. 359-369. DOI: 10.1016/j.msea.2014.01.024 
3. Mechanical behavior of $\mathrm{SiC}$ foam-SiC particles/Al hybrid composites / Long-Zhi Zhao, Ming-Juan Zhao, Hong Yan, Xiao-Ming Cao, Jin-Song Zhang // Trans. Nonferrous Met. Soc. China. - 2009. - Vol. 19. - P. 547-551. - DOI: 10.1016/S10036326(10)60106-9

4. Ortega-Celaya F., Pech-Canul M. I., Lopes-Cuevars J., Rendon-Angeles J. C., Pech-Canul M. A. Microstructure and impact behavior of $\mathrm{Al} / \mathrm{SiCp}$ composites fabricated by pressure less infiltration with different types of SiCp // Mater. Proces. Techn. - 2007. - Vol. 183. - Nos. 2-3. P. 368-373. - DOI: 10.1016/j.jmatprotec.2006.10.029

5. Коновалов А. В., Смирнов С. В. Современное состояние и направления исследований металломатричных композитов $\mathrm{Al} / \mathrm{SiC} / /$ Конструкции из компо- зиционных материалов. 2015. - № 1. - C. 30-35.

6. Pugacheva N. B., Michurov N. S., Bikova T. M. The Structure and Properties of the 30Al-70SiC Metal Matrix Composite Material // Diagnostics, Resource and Mechanics of materials and structures. - 2015. - Iss. 6. -P. 6-18. - URL: http://dreamjournal.org/issues/2015-6/20156 56.html 11

7. Pugacheva N. B., Michurov N. S., Bykova T. M. Structure and properties of the Al/SiC composite material // Physics of Metals and Metallography. - 2016. - Vol. 117, no. 6. C. 634-640. - DOI: 10.1134/S0031918X16060119

8. Smirnov A. S., Konovalov A. V., Muizemnek O. Yu. Modelling and Simulation of Strain Resistance of Alloys Taking into Account Barrier Effects // Diagnostics, Resource and Mechanics of materials and structures. - 2015. - Iss. 1. - P. 61-72. - URL: http:// dream-journal.org/issues/ /2015-1/2015-1_18.html

9. Халевицкий Ю. В., Мясникова М. В., Коновалов А. В. Приемы создания вычислительной модели представительных объемов металломатричного композита Al/SiC с внутренней структурой // Математическое моделирование в естественных науках. - 2014. - Т. 1. C. 277-280.

10. Колачев Б. А., Елагин В. И., Ливанов В. А. Металловедение и термическая обработка цветных металлов и сплавов. - М. : Изд-во МИСИС, 2001. - 416 с.

11. Fridlyander I. N. Modern Aluminum and Magnesium Alloys and Composite Materials Based on Them // Metal Science and Heat Treatment. - 2002. - Vol. 44. - Iss 7. - P. 292-296. DOI: $10.1023 / \mathrm{A}: 1021255804324$

12. Phase Stability and Interface Reactions in the Al-SiC System / Doh-Jae Lee, M. D. Vaudin, C. A. Handewerker, U. R. Katter // Mater. Res. Symp. Proc. - 1988. - Vol. 120. - P. 293. DOI: $10.1557 /$ PROC-120-357

13. Ibrahim A. I., Mohamed F. A., Lavernia E. J. Particular reinforced metal matrix composites a review // Journal of Materials Science. - 1991- Vol. 26 - P. 1137-1156. - DOI: 10.1007/BF00544448

14. Structure and thermophysical properties of aluminum-matrix composites / N. B. Pugacheva, N. S. Michurov, E. I. Senaeva // Physics of Metals and Metallography. - 2016. - No. 11. - P. 11441151. - DOI: 10.1134/S0031918X16110119 